\title{
Investigation of passive edge emission in charge exchange spectra at the ASDEX Upgrade tokamak
}

\author{
E. Viezzer, T. Pütterich, R. Dux, A. Kallenbach and the \\ ASDEX Upgrade Team \\ Max-Planck-Institut für Plasmaphysik, EURATOM Association, Boltzmannstr. 2, \\ 85748 Garching, Germany \\ E-mail: Eleonora.Viezzer@ipp.mpg.de
}

\begin{abstract}
The influence of passive edge emission on the charge exchange spectra of carbon $\left(\mathrm{C}^{5+}, \mathrm{n}=8 \rightarrow 7\right.$ at $\left.529 \mathrm{~nm}\right)$ measured in fusion plasmas at the ASDEX Upgrade tokamak is investigated. The spectra are obtained viewing the plasma edge tangentially with 8 lines of sight while the plasma is swept to enhance the spatial density of the measurements. A forward model to deconvolute the measured line-integrals is employed. The local emissions are then compared to the simulated radiation obtained with the 1-D impurity transport code STRAHL using transport coefficients which are determined independently. Depending on the background neutral deuterium densities the simulation predicts the absolute line intensities and the relative contributions of electron impact excitation and thermal charge exchange to the measured signals. Therefore, a background neutral deuterium density profile has been determined. For the passive emission line of $\mathrm{C}^{5+}$, the comparison between forward model and simulations yields that electron impact excitation and thermal charge exchange are both important for understanding the passive line. Indeed, thermal charge exchange proves to be affecting the passive emission line considerably via two mechanisms, i.e. change of ionization equilibrium through charge exchange recombination and radiation due to local charge exchange reactions.
\end{abstract}

\section{Introduction}

Active charge exchange recombination spectroscopy (CXRS) relies on a neutral beam providing energetic neutrals which perform a charge exchange (CX) reaction with fully ionized impurity ions [1]. This technique allows a derivation of ion temperature, density and velocity profiles. The challenge hereby is that the CXRS measurements are superimposed complex spectra consisting of an active spectrum and a background passive emission spectrum. Therefore, the analysis and the interpretation of the spectra require the distinction of both active and passive line emission. For CXRS measurements at ASDEX Upgrade viewing the beam in the plasma core, the passive edge emission plays a major role due to the attenuation of the neutral beam as it penetrates the plasma. Therefore, the separation of the two components is mandatory. At the plasma edge, the 
amplitude of the passive signal is significantly lower than the active one allowing to apply rough models for the passive component.

The deconvolution of the line-of-sight (LOS) integrated, passive measurements allows the determination of the radial emissivity distribution of the measured spectral lines. Analyzing the radial profile helps to quantify the passive emission lines and improves the evaluation of the superposed active CX spectra. The quantitative evaluation of the passive component is important not only for understanding and interpreting the active spectra but can also provide information on the background neutral density at the plasma edge. This on the other hand helps to understand the plasma behaviour, especially the fueling of the plasma.

This paper is organized as follows. In section 2 a brief overview of the CXRS technique is given. The deconvolution of the line-integrated measurements is presented in section 3, while in chapter 4 the impact of the neutrals is described. In addition, the derivation of a local neutral deuterium (D) density profile and a flux surface averaged $\mathrm{n}_{D}$ profile is discussed. The results of modelling the passive carbon emission are presented in section 5. An application of the forward model, i.e. the reconstruction of measured spectra, is given in section 6 .

\section{Measurement technique}

CXRS [1, 2, 3] has become a powerful diagnostic tool in magnetically confined fusion plasmas which provides spatially and temporally resolved measurements of ion temperatures and velocities from Doppler widths and shifts. The technique exploits spectral lines which are produced by charge transfer from hydrogen $(\mathrm{H})$ or deuterium (D) atoms into excited states of impurity ions, i.e. from the $\mathrm{CX}$ reaction $\mathrm{A}^{Z+}+\mathrm{D}^{0} \rightarrow$ $\left(\mathrm{A}^{(Z-1)+}\right)^{*}+\mathrm{D}^{+}$. The recombined ion is born in an excited state and during the subsequent decay it emits photons at characteristic wavelengths. Those of a certain wavelength are collected via CXRS.

Neutral atoms (H or D) are injected into the plasma via neutral beam injection (NBI). Along the NBI trajectory a local CX signal can be detected leading to a spatially localized measurement since the detected impurity ions radiate in the region where the chords of the CXRS diagnostic intersect the path of the neutral beam particles. CX processes occur also between fully ionized impurities and neutral background atoms, which enter the plasma from the walls and represent localized sources of neutrals, leading to a passive or thermal CX emission line $[4,5]$. Most of the background neutrals are ionized at the very edge of the plasma, however, a few transfer their charge after a CX reaction. An additional background emission results from electron impact excitation processes which also occur at the plasma edge.

The measured spectra are line-integrated and a composite of signals due to electron impact excitation, $\mathrm{S}_{e x c}$, recombination followed by photon emission, $\mathrm{S}_{r e c}$, thermal CX, $\mathrm{S}_{t c x}$ and the NBI-induced active CX signal, $\mathrm{S}_{a c x}$. Hence, the total signal is $\mathrm{S}_{t o t}=\mathrm{S}_{e x c}$ $+\mathrm{S}_{r e c}+\mathrm{S}_{t c x}+\mathrm{S}_{a c x}=\mathrm{S}_{\text {pass }}+\mathrm{S}_{a c x}$. In this work, the passive signal $\mathrm{S}_{\text {pass }}[4,6,7]$ of 
carbon $\left(\mathrm{C}^{5+}, \mathrm{n}=8 \rightarrow 7\right.$ at $\left.529 \mathrm{~nm}\right)$ which is localized in the vicinity of the edge transport barrier (ETB) [8] has been investigated with a special emphasis on $\mathrm{S}_{e x c}$ and $\mathrm{S}_{t c x}$. For the passive emission of $\mathrm{C}^{5+}$ two charge states are contributing. Electron impact excitation operates on the $\mathrm{C}^{5+}$ state, while charge exchange with thermal neutrals operates on the $\mathrm{C}^{6+}$ state. At the plasma edge, the plume effect [1] caused by excited $\mathrm{C}^{5+}$ ions drifting through the LOS is small and therefore negligible.

\subsection{Edge CXRS diagnostic at ASDEX Upgrade}

At the ASDEX Upgrade tokamak [9], currently one diagnostic for CX studies of the plasma edge is installed. It is located in the outer midplane, at the low-field side (LFS) of the vessel and has been designed in order to achieve high spatial resolution (up to 3 $\mathrm{mm})$. A detailed description of the diagnostic can be found in $[10,11]$. On the left-hand side of figure 1 a poloidal cross-section of ASDEX Upgrade is illustrated indicating the poloidal location of the edge CXRS diagnostic. Concerning the passive emissions the

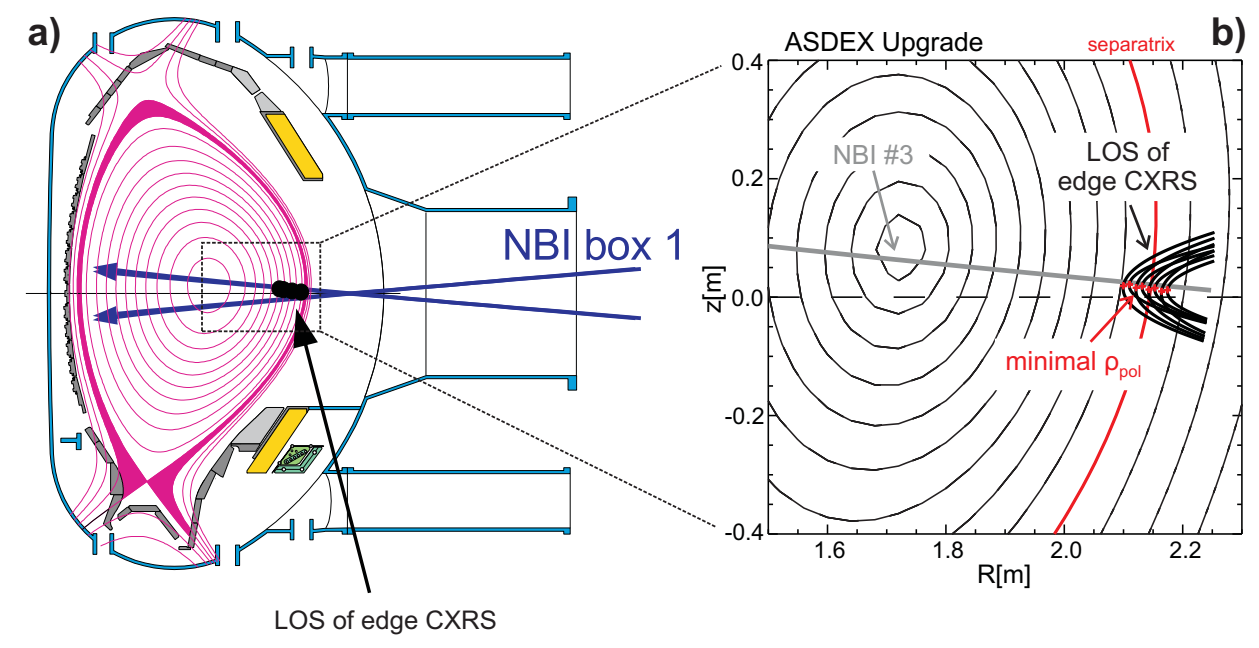

Figure 1. a) Poloidal cross-section of ASDEX Upgrade and location of chords of edge CXRS diagnostic, b) overview plot of minimal $\rho_{\text {pol }}$ coordinate.

system provides LOS-integrated measurements and does not give any local information at a specific radial position. Therefore, all line-integrated plots which result directly from the measurements are given as a function of the minimal $\rho_{\text {pol }}$ coordinate; $\rho_{\text {pol }}$ being the normalized poloidal flux label touched by the viewing chord (see figure $1 \mathrm{~b}$ )).

The passive spectral line is usually fitted by one Gaussian function and by integrating the Gaussian component over the wavelength the radial distribution of the cold edge emission can be obtained. Figure 2 a) shows a passive spectrum of $\mathrm{C}^{5+}$ measured in an H-mode [8] plasma. In figure $2 \mathrm{~b}$ ) a profile of the brightness of $\mathrm{C}^{5+}$ is illustrated. Each data point corresponds to one measured spectrum. The radial density of the data points is achieved by making a radial sweep of the plasma in order to cover the whole edge pedestal. In order to obtain a local emissivity profile a forward model 


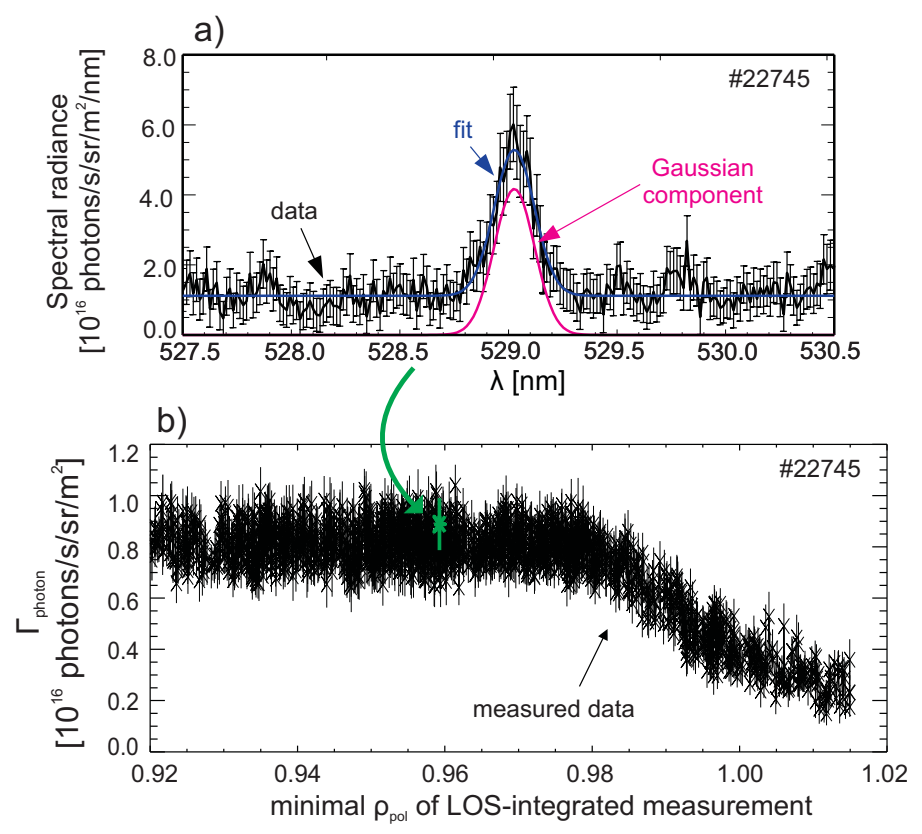

Figure 2. a) Passive spectrum of $\mathrm{C}^{5+}$ measured in type-I ELMy H-mode discharge \#22745 with corresponding fit (in blue) and Gaussian component of fit (in magenta), b) resulting photon flux.

has been developed which deconvolutes the LOS-integrated measurements (see section $3)$.

It may be noted that the standard procedure of the CXRS evaluation for identifying the active CX component uses either an estimate of the passive emission temperature or beam modulation, i.e. the alternating switching-on and switch-off of the neutral beam. Beam modulation allows a measurement of the active and passive signal within short consecutive time intervals. The active component is obtained by subtraction. For suppressing the passive background features the beam modulation technique is routinely applied at ASDEX Upgrade. However, in the case when the ionization equilibrium is affected by switching on the diagnostic beam and the background neutral density is changed compared to the beam-off phase, this technique fails [12]. Hence, a tool which characterizes the passive background line emission and does not rely on beam modulation helps to quantify the passive components in an active CX spectrum.

\section{Deconvolution of line-integrated measurements}

A forward model for deconvoluting the line-integrated measurements has been developed. The model function for the radial emissivity consists of a modified Gaussian, which is a combination of two Gaussian functions with different decay lengths. The center of emission is expected to be around the separatrix in the edge region. To the left-hand of the center, i.e. towards the plasma core, a Gaussian function with a certain width is assumed and to the right-hand (towards the scrape-off layer (SOL) of the plasma) a Gaussian with different width. Both combined yield the model 

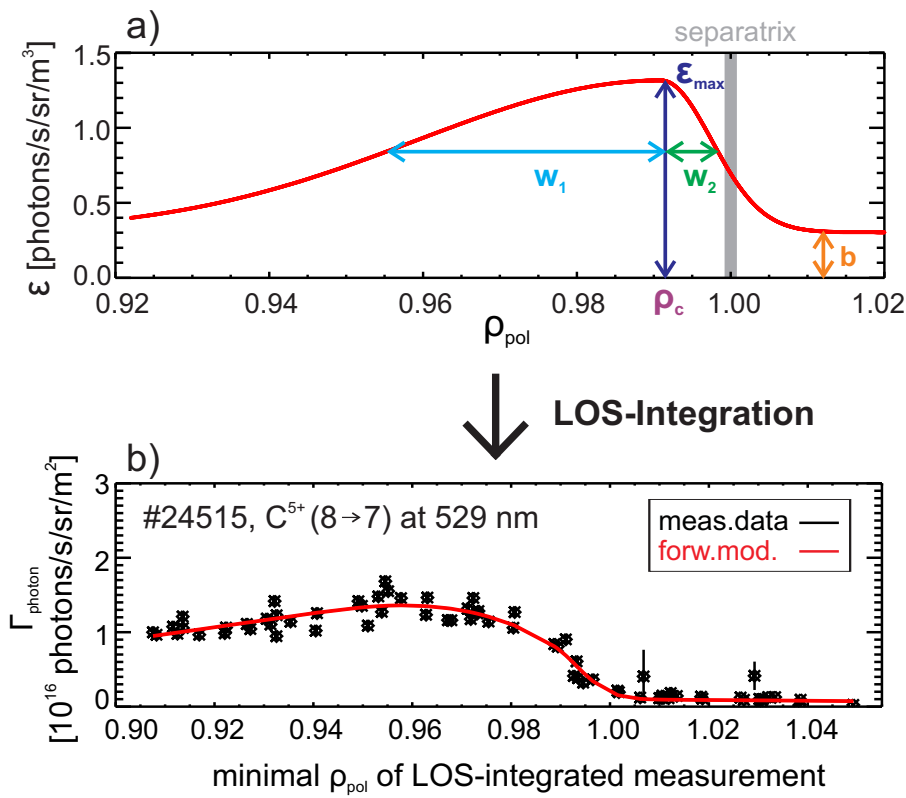

Figure 3. Forward modelling of passive edge emission: a) fitting parameters of model function, b) measured photon flux of $\mathrm{C}^{5+}$ in black (obtained in type-I ELMy H-mode discharge \#24515) and modelled line-integrated intensity in red.

function. The fitting parameters besides the two Gaussian widths $\mathrm{w}_{1}$ and $\mathrm{w}_{2}$ are the maximum and center of emission, $\epsilon_{\max }$ and $\rho_{c}$ respectively, and a parameter $\mathrm{b}$ which accounts for the background possibly caused by diffuse reflection. Note that a possible bremsstrahlung contribution is excluded as the radial distribution of the passive emission line is obtained by integrating the Gaussian component of the fitted spectral line. Figure 3 a) shows the fitting parameters of the forward model which describe the local emissivity profile. The fit parameters are adjusted iteratively by a gradient-expansion algorithm, which calculates a non-linear least-squares fit, until the resulting LOS-integrated profile describes the measurement best, see figure $3 \mathrm{~b}$ ).

To interpret the passive emission in terms of a physics model, the radiation is simulated with the 1-D particle transport code STRAHL $[13,14]$, which evaluates the impurity behaviour in axisymmetric magnetic configurations, such as ASDEX Upgrade. STRAHL calculates the radial transport and the impurity emission in the plasma edge by solving the coupled radial transport equations (averaged over the magnetic flux surfaces) for each ionization stage of an impurity ion. After the particle density and the ion stage distribution (in which also the total CX rates and the input background neutral deuterium densities enter) are computed, STRAHL makes a post-processing calculation of the radiation including emissivity profiles due to the total line radiation of each ionization stage, bremsstrahlung and radiation from recombination.

Electron density and temperature are input parameters and are derived from the measurements of the electron cyclotron emission (ECE) diagnostic [15], the Thomson scattering system [16], impact excitation spectroscopy at a Lithium beam [17, 18] and laser interferometry [19], respectively. In STRAHL, the impurity transport is 
treated using a flux surface averaged diffusion coefficient and a drift velocity profile as determined by active CXRS measurements. The active CX spectra are measured in a discharge with the same setup but in addition, a neutral beam viewed by the diagnostic is switched on while another heating beam outside the view of the CXRS system is switched off. The transport model also takes the effects of Edge Localized Modes (ELMs) [20, 21] into account. Due to the occurrence of ELMs, which eject plasma and impurity ions from the magnetically confined plasma, a quasi-equilibrium of the density is set up. The quasi-equilibrium of the impurity density depends on the transport coefficients and on the influx of the considered impurity ion, i.e. knowledge of the total density evolution of the impurity species yields information on the transport coefficients by using independent evaluation methods. Comparing the modelled impurity density profile to the measured one, which can be obtained with CXRS, the best suited transport coefficients can be calculated via a forward model [22]. The simulations of the passive emission lines are fixed by the transport coefficient profiles as well as by the impurity content, parameters which result from the analysis of the active CX spectra. At the ETB, the diffusive transport in-between ELMs is low and a strong inward drift is observed, while the effect of an ELM is implemented by increasing D to an anomalously high value. A detailed description on the evaluation of $\mathrm{D}$ and $\mathrm{v}$ in the ETB can be found in [22]. The influx of carbon is adjusted to match the measured $\mathrm{C}^{6+}$ density at the pedestal. It is anticipated that the densities of $\mathrm{C}^{5+}$ are rigidly coupled to those of $\mathrm{C}^{6+}$, as the maximum of $\mathrm{C}^{5+}$ is inside the separatrix.

Modelling the passive edge emission requires various atomic data, which are derived from the Atomic Data Analysis Structure (ADAS) [23]. In detail, electron impact excitation rate coefficients and CX rate coefficients specific for photon emission, total CX rate coefficients, ionization and recombination rate coefficients are needed. Neutral $\mathrm{D}$ is present in the ground state as well as in excited states, both contributing to the CX process. It should be noted that the major contribution to all thermal CX processes is provided by $n=2$ of $D$ and it is not expected that higher states than $n=4$ of D play a significant role. This is in agreement with the findings in [24]. In order that the transport model considers the excited states of $\mathrm{D}$, modifications of the atomic data had to be made since STRAHL simulates thermal CX reactions by taking the according rate coefficients and the radial neutral density, which is an input parameter, into account. A set of effective CX coefficients was derived from the ADAS database taking a collisional-radiative model for D into account. The atomic data are calculated such that the distribution of the ground and excited states of D is included and the CX cross-sections are combined accordingly, i.e. the effective CX rate coefficients are weighted by the relative abundance of $\mathrm{D}$ in $\mathrm{n}=1,2, \ldots$ state. The data set is compiled using $\mathrm{T}_{e}=\mathrm{T}_{i}=\mathrm{T}_{n}$ which is often assumed if no detailed information about $\mathrm{T}_{i}$ and $\mathrm{T}_{n}$ is available. For the CX processes, two types of atomic data are of interest: the total recombination coefficients, i.e. rate coefficients which refer to CX recombination, and rate coefficients for photon emissions following a CX reaction. These so-called photon emissivity coefficients (PECs) are effective rate coefficients, which take into account 
electron impact excitation, cascades and additional populating processes. Within this work PEC-EIE represents electron impact excitation rate coefficients specific for photon emission, while PEC-CX are the rate coefficients for photon emission after a CX process. The PECs are calculated from Maxwell averaged rate coefficients of the specific process multiplied by the branching ratio $\mathrm{B}$ which defines the relative probability of the transition.

In order to calculate the rate coefficients accurately, both ground and excited states of D have been considered via:

$$
\begin{aligned}
& R_{C X}=\sum_{j=1}^{n_{\max , D}} n_{D_{j}}\left(n_{e}, T_{e}\right) n_{I, Z} Q_{j}\left(T_{i}, T_{n}\right) \\
& \epsilon_{C X, k \rightarrow m}=\sum_{j=1}^{n_{\max , D}} n_{D_{j}}\left(n_{e}, T_{e}\right) n_{I, Z} q_{j, k}\left(T_{i}, T_{n}\right) B_{k \rightarrow m}
\end{aligned}
$$

where $\mathrm{R}_{C X}$ represents the recombination rates per volume and $\epsilon_{C X}$ the photon rates per volume. For both formulas, the $\mathrm{n}$ states of $\mathrm{D}$ are summed up. For CX between carbon and a thermal neutral atom the Maxwell averaged rate coefficients $\left(\mathrm{Q}_{j}\right.$ and $\mathrm{q}_{j, k}$, respectively), which depend on ion temperature $\mathrm{T}_{i}$ and neutral temperature $\mathrm{T}_{n}$, are available up to $\mathrm{n}=4$ for the donor $\mathrm{D}$. The density of each state of deuterium $\mathrm{n}_{D_{j}}$ is given by the total deuterium density $\mathrm{n}_{D}$ and the fractional abundance of each state $f_{j}$ :

$$
n_{D_{j}}\left(n_{e}, T_{e}\right)=n_{D} \cdot f_{j}\left(n_{e}, T_{e}\right)
$$

$f_{j}$ depends on electron density and temperature and is calculated from a collisionalradiative model for $\mathrm{D}$, which accounts for dominant excitation processes and for the specific Einstein coefficients of each state. These are derived from [25]. In equation (1), $\mathrm{Q}_{j}$ is the total rate coefficient referring to CX processes, while in equation (2) $\mathrm{q}_{j, k}$ provides for the partial rate coefficients of the CX reactions. $\mathrm{q}_{j, k}$ specifies the rate coefficients from each $\mathrm{n}$ state of $\mathrm{D}$ to each $\mathrm{n}$ state of the receiver. These are effective coefficients taking the population of state $\mathrm{k}$ through cascades into account. The parameter $\mathrm{B}_{k \rightarrow m}$ in equation (2) represents the branching ratio for a specific transition. Note that in ADAS the rate coefficients for CX between neutral atom and impurity ion are available only for the donor H. At ASDEX Upgrade, deuterium (D) performs the CX reaction, however, no differences are expected between $\mathrm{H}$ and $\mathrm{D}$ cross-sections at identical velocity.

\section{Impact of neutrals}

For simulating the local emissivity due to thermal CX, two different effects have to be taken into account, i.e. CX recombination which affects the ion stage distribution and direct excitation due to local CX reactions. The effect of $\mathrm{CX}$ recombination on the local ionization balance is as follows: the equilibrium between ionization and recombination is controlled by electron impact ionization and by radiative and dielectronic recombination. CX recombination constitutes an additional source on the 
flux surface and has an effect on the ionization-recombination balance, i.e. on the distribution of the relative abundance of the neutral stages and all ionization stages of the impurity ion. For carbon, CX recombination represents an additional $\mathrm{C}^{5+}$ source, i.e. the density of $\mathrm{C}^{5+}$ is increased and hence, the intensity of the passive emission line excited by electron impact excitation of $\mathrm{C}^{5+}$ is increased. The magnitude of this effect depends strongly on the penetration of the background neutrals into the plasma.

The ionization balance between $\mathrm{C}^{6+}$ and $\mathrm{C}^{5+}$ is a global quantity, i.e. it is constant on the flux surface since the characteristic time for equilibration between the ion stages is long against the time to distribute on the flux surface. The equilibration time, which is given by the ionization time, is much larger than the transit time. The transit time of a passing particle is the time it needs to travel from the outside to the inside of the torus. In the investigated discharges the transit time is in the range of $10^{-5}-10^{-6} \mathrm{~s}$ and the time scale for a particle to be ionized is within ms. As the background neutral atoms are subject to a toroidal asymmetry, it is required to account for a global or flux surface averaged neutral D density when considering a change of the ionization balance through CX recombination.

However, for the photon emissivity after CX, the local D density has to be taken into account. Here, the time of a photon to be released after a CX reaction, i.e. the emission time, is much smaller than the transit time. For the $\mathrm{H}$-like ion $\mathrm{C}^{5+}$ the emission time is in the range of several ns.

For the present analysis of the passive $\mathrm{C}^{5+}$ emission, the change of the ionization equilibrium through CX recombination accounts for approximately $15 \%$ to the local brightness emitted in the narrow ETB layer. Direct excitation due to local CX reaction contributes around 55\%. The remaining $30 \%$ are emitted due to electron impact excitation.

\subsection{Deduction of neutral deuterium density profile}

The mechanisms of the interaction between neutrals and the plasma are mainly ionization and CX, however, also atomic excitation and molecular dissociation are important processes, since neutrals are present in the atomic as well as in the molecular state. In order to understand the penetration of the neutrals, the source of neutral particles has to be established. Neutral particles exist mainly due to plasma recycling, i.e. the interaction between the plasma and the wall of the vacuum vessel [26], and fueling by gas puffs at specific locations. Ions which are neutralized during wall collision constitute a source of atoms which almost have the same energy as the ions which are reflected at the surface of the wall. Via ion impact the atoms can be released and penetrate into the plasma. In addition, also molecules can be released leading to a contribution of both atoms and molecules [27]. In this work, the recycling pattern of the vacuum chamber is divided into four regions, i.e. the outboard limiters at the lowfield side (LFS), the heatshield at the high-field side (HFS) and the upper and lower divertor, covering the main recycling locations of ASDEX Upgrade [28]. 


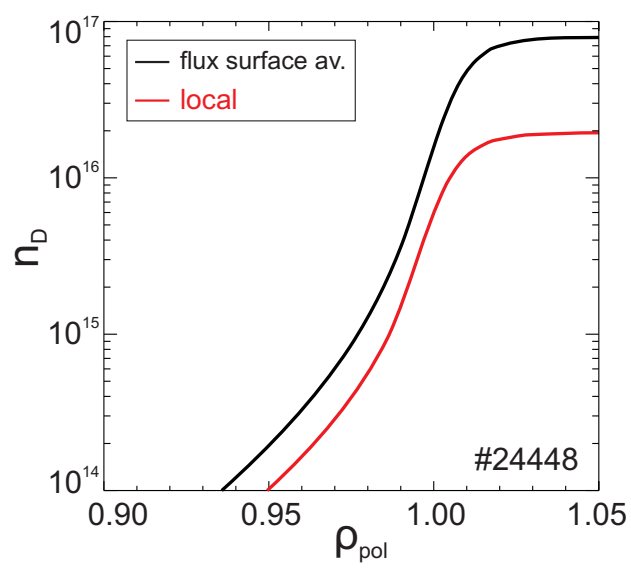

Figure 4. Neutral D density profiles resulting from modelling using KN1D (input profiles derived from measured data in \#24448): $\mathrm{n}_{D}$ for local CX reactions in red, while in black flux surface averaged $\mathrm{n}_{D}$, i.e. averaged in toroidal and poloidal direction.

Since there are no direct measurements for the $\mathrm{n}_{D}$ profiles, they have been simulated with the kinetic transport model KN1D [29]. The 1-D in space and 2-D in velocity kinetic transport algorithm KN1D calculates the distribution functions of neutral atomic and molecular $\mathrm{D}$ using a method of successive collision generations with specified input plasma parameters, such as $\mathrm{n}_{e}, \mathrm{~T}_{e}$ and $\mathrm{T}_{i}$ profiles. In addition, a molecular neutral wall pressure, which is derived from measured neutral flux densities at ASDEX Upgrade, is specified in the input file. Within this framework, the input profiles for the simulations are derived from the measured data obtained in a type-I ELMy H-mode discharge (\#24448) with low ELM frequency of about $60 \mathrm{~Hz}$. The measurements are ELMsynchronized, i.e. the measured data are selected relatively to the occurrence of ELMs to extract the inter-ELM phase. For the local CX processes, i.e. at the location of the CXRS measurements, a neutral D density profile has been derived (see red curve in figure 4). The neutral pressure is measured by a manometer in the NBI port, i.e. at the toroidal location where photons resulting from local CX reactions are detected by the chords of the edge CXRS diagnostic. However, for a change of the ionization balance through CX recombination the $3-\mathrm{D}$ distribution of the neutrals has to be considered. Therefore, an estimate of the toroidally and poloidally averaged $n_{D}$ profile, which corresponds to a flux surface averaged $n_{D}$ profile (see black curve in figure 4), has been derived. To this end, the geometry of the flux surfaces at the different positions and the varying neutral wall pressures at different toroidal and poloidal locations have been taken into account. Following assumptions have been made to estimate the flux surface averaged $n_{D}$ profile:

(i) At the HFS, as well as for the upper and lower divertor, there are no measurements of $\mathrm{n}_{e}$ and $\mathrm{T}_{e}$ profiles. Hence, $\mathrm{n}_{e}$ and $\mathrm{T}_{e}$ measured at the LFS have been mapped accordingly.

(ii) The physics of the SOL, such as the SOL transport, has not been implemented and the details of $\mathrm{n}_{e}$ and $\mathrm{T}_{e}$ profiles in the SOL have not been taken into account. An exponential decay of both profiles was assumed. 


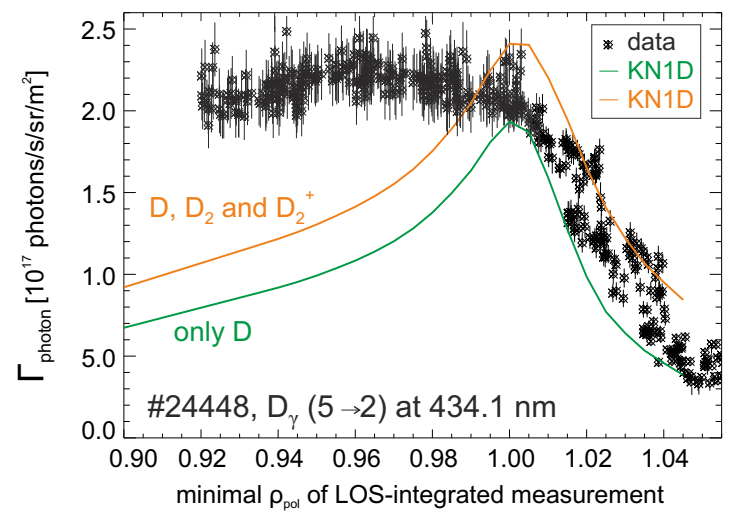

Figure 5. Comparison of photon flux derived from KN1D simulations (green and orange) to $\mathrm{D}_{\gamma}$ measurement (black data points) obtained in type-I ELMy H-mode discharge \#24448. The deconvolution of the measured data inside the separatrix is not possible due to the toroidal asymmetric distribution of the background neutrals.

Since background neutrals mainly exist at the HFS of the ASDEX Upgrade tokamak, i.e. at the inner heatshield and lower divertor baffle, due to main chamber recycling, the poloidal average of $\mathrm{n}_{D}$ is mostly affected by these two parts. For simulating the penetration of the neutrals at the heatshield of the HFS, the neutral wall pressure has been derived from the $\mathrm{D}_{\epsilon}(\mathrm{n}=7 \rightarrow 2)$ photon flux measured by visible spectroscopy. However, the measured intensities might be hampered by reflections from the divertor regions (due to the tungsten coated plasma facing components at ASDEX Upgrade) leading to an overestimation of $n_{D}$ at the HFS. Hence, the total averaged $n_{D}$ profile might be overrated. Another source of error results from the input neutral wall pressure, which is measured by the neutral gauge manometers at remote positions. The calibration error of the gauge manometers at ASDEX Upgrade is $10-15 \%$. As the ionization gauges are available only at a few distinct toroidal and poloidal locations, the flux surface averaged $\mathrm{n}_{D}$ profile has been extrapolated, which leads to uncertainties of the total averaged $\mathrm{n}_{D}$ profile as well.

The modelled local influx of the background neutrals can be compared to the $\mathrm{D}_{\gamma}$ $(\mathrm{n}=5 \rightarrow 2$ at $434.1 \mathrm{~nm})$ photon flux measured by the edge CXRS diagnostic in the type-I ELMy H-mode discharge \#24448. However, since the background neutrals are subject to a toroidal asymmetry, the deconvolution of the $\mathrm{D}_{\gamma}$ measurements fails especially for chords viewing the confined plasma inside the separatrix. Figure 5 shows the lineintegrated $\mathrm{D}_{\gamma}$ measurements, while the photon flux derived from the simulated local $\mathrm{n}_{D}$ profile is illustrated in green. The green curve represents the atomic contribution of $\mathrm{D}$, while the orange curve also includes the molecular contribution of $\mathrm{D}_{2}$ and $\mathrm{D}_{2}^{+}$. Note the additional effect of the molecules, which mainly exist in the SOL, on the line-integrated profile. The modelled photon flux results from the LOS-integration of the local emission, which can be calculated directly from $\mathrm{n}_{D}$ accounting also for the specific PECs of the transition. Only electron impact excitation is considered for the emission of photons. According to the atomic data stored in ADAS the contribution of thermal CX to the 
passive emission line of $\mathrm{D}_{\gamma}$ is low since recombination excites predominantly states $\mathrm{n} \leq$ 4. Note that for the outer chords a fair agreement is found. The measurements might yield a higher photon flux due to the possible contribution of the molecules.

For spectroscopic flux measurements the quantity S/XB [30] is of high importance as it allows an interpretation of measured photon fluxes in terms of particle fluxes. $\mathrm{S} / \mathrm{XB}$ is the ratio of probabilities due to ionization $(\mathrm{S})$ and excitation $(\mathrm{X})$ including the branching ratio $\mathrm{B}$ and represents an inverse photon efficiency. Modelling of the background neutrals yields an effective $\mathrm{S} / \mathrm{XB}$ of 18.2 , which agrees reasonably well with the commonly accepted value of about 15 for $\mathrm{D}_{\alpha}$ for typical edge conditions $[31,32]$.

\section{Experiment and Results}

The passive spectral line of $\mathrm{C}^{5+}(\mathrm{n}=8 \rightarrow 7$ at $529 \mathrm{~nm})$ was measured in the type-I ELMy H-mode discharge \#24515. The forward model for the radial profile of the passive emission was applied to the data to derive the local emissivity profile of $\mathrm{C}^{5+}$ (see red profile in figure 6). The measured data are ELM-filtered, i.e. the data recorded during the occurrence of an ELM are sorted out and only data in-between ELMs are plotted. The carbon density does not exhibit a considerable evolution between the ELMs as discussed in [22].

The results of the forward model are then compared to the simulated radiation obtained with the 1-D code STRAHL. In figure 6 the curves in dark blue result

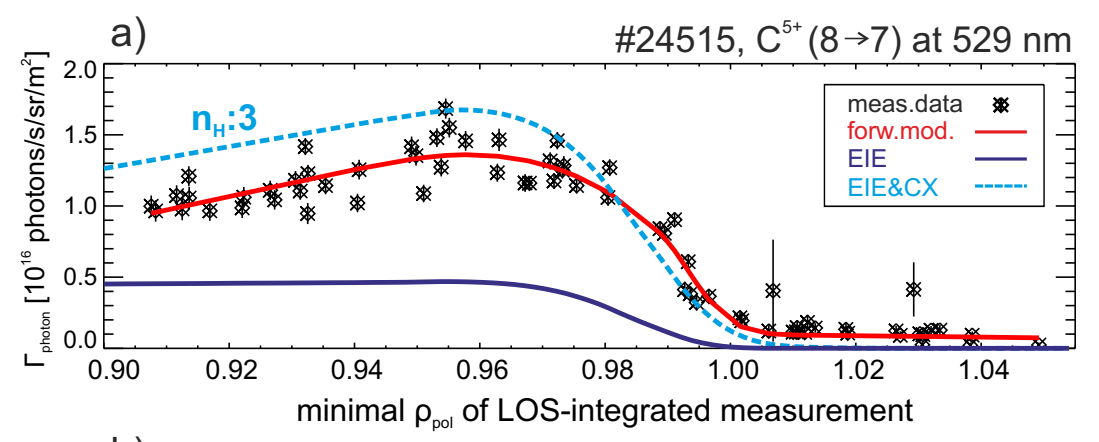

b)

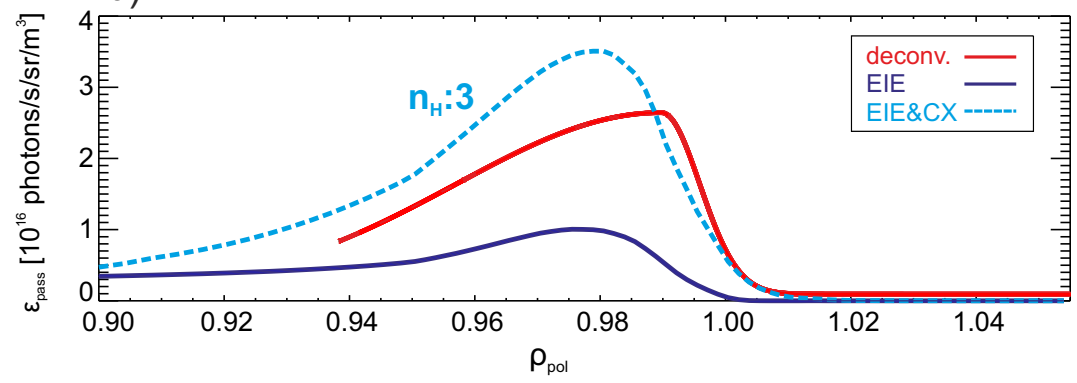

Figure 6. a) Photon flux and b) deconvoluted emissivity profile of $\mathrm{C}^{5+}$ : measured data in black (obtained in type-I ELMy H-mode discharge \#24515), forward model in red, STRAHL simulations in blue (dark blue corresponding to radiation due to electron impact excitation, while light blue (dashed line) accounts for electron impact excitation and thermal CX). 
from simulating electron impact excitation processes only. Note that electron impact excitation is not sufficient to explain the emitting source of the passive emission line of $\mathrm{C}^{5+}$. Besides not providing sufficient emissivity, the shape is different and does not describe the shape of the measured emissivity (in red). Even if the atomic data of electron impact excitation would be underestimated, the shape would still not match. The local emissivity is linearly dependent on the atomic data for electron impact excitation, i.e. raising the PEC-EIE results in a linear increase of the emissivity. The modelled emissivity which results from electron impact excitation only is rather constant up to $\rho_{\text {pol }} \leq 0.96$, increases and reaches its maximum at $\rho_{\text {pol }} \approx 0.98$. The gradient near the separatrix is rather flat compared to the gradient of the measured emissivity profile. A second source of light, which provides for the steep gradient at the separatrix is needed. The inclusion of thermal CX leads to an increase of the local emissivities (see light blue curves in figure 6) and a better fit to the shape of the measured data. For the thermal CX simulation in figure 6 both neutral D density profiles modelled with KN1D (shown in figure 4) have been included, i.e. for the change of the ionization equilibrium through CX recombination the flux surface averaged $\mathrm{n}_{D}$ profile has been used while for direct excitation due to thermal CX reactions the local $n_{D}$ has been implemented. Both $\mathrm{n}_{D}$ profiles have been divided by a factor of 3 in order to get a good agreement to the measured emissivity profile. Using the modelled $\mathrm{n}_{D}$ profiles as they result from the KN1D simulations would overestimate the passive $\mathrm{C}^{5+}$ emission by a factor of 2.5 . Note that photon emission after a CX reaction is not fully linear to the neutral density due to the additional effect of CX recombination on the ionization balance.

For modelling thermal CX reactions, the penetration of the neutrals into the plasma is decisive and the contribution of CX depends strongly on the background neutral
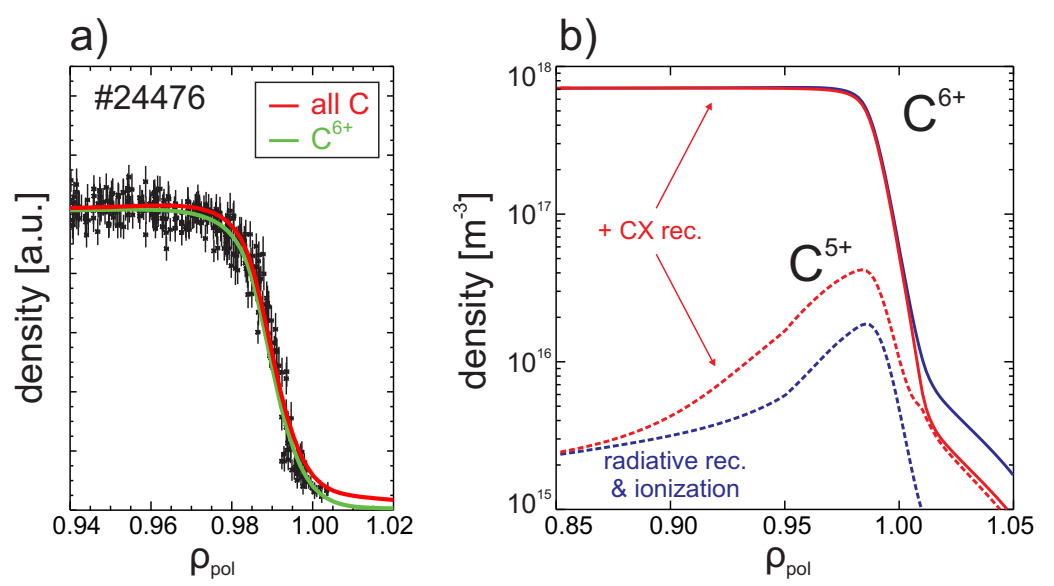

Figure 7. a) Measured $\mathrm{C}^{6+}$ density profile along with best fit (in green) derived from modelling the active CXRS measurements [22], while the red curve taken from the same model represents the total carbon density profile; b) change of the ionization equilibrium due to thermal $\mathrm{CX}$ : ion stage distribution of $\mathrm{C}^{5+}$ and $\mathrm{C}^{6+}$ due to radiative recombination and ionization in blue, while in red the additional effect of CX recombination is visible. 
density profiles. In the case of carbon, CX recombination reduces slightly the abundance of fully ionized $\mathrm{C}^{6+}$ but raises at the same time the abundance of $\mathrm{C}^{5+}$ (as can be seen in figure 7) leading to an increase of the passive $\mathrm{C}^{5+}$ emission. Here, the 3-D density distribution of the background neutrals plays a crucial role. An increase of $n_{D}$ leads to a higher abundance of $\mathrm{C}^{5+}$, which is born naturally due to the additional effect of $\mathrm{CX}$ recombination on the flux surface, and causes therefore a higher intensity of $\mathrm{C}^{5+}$ and vice versa. Hence, the ion stage distribution due to CX recombination is strongly dependent on the global penetration of the neutrals into the plasma. On the other hand, radiation due to local CX reactions has to be accounted for. For that the photon emissivity coefficients PEC-CX are used. In figure 7 a) the measured $\mathrm{C}^{6+}$ density profile along with the modelled profile which describes the measured data best (in green) is illustrated. The total density profile of carbon is shown in red. The fit results from the analysis of the active CX spectra [22]. Figure $7 \mathrm{~b}$ ) shows the effect of CX recombination, i.e. the redistribution of the ion stages. Note that CX recombination provokes an increase of the fractional abundance of $\mathrm{C}^{5+}$ and in addition, also a slight shift of the maximum towards smaller radii.

Both effects of CX can be modelled separately by calculating the ionization balance with and without the total recombination coefficients. The contribution of photon emission due to local CX processes can be quantified by simulating the radiation including the PEC-CX. Separating both effects of CX in the simulations (see figure 8) yields that considerable photon emission results from both CX contributions. All simulations account for electron impact excitation, which is shown in dark blue in figure

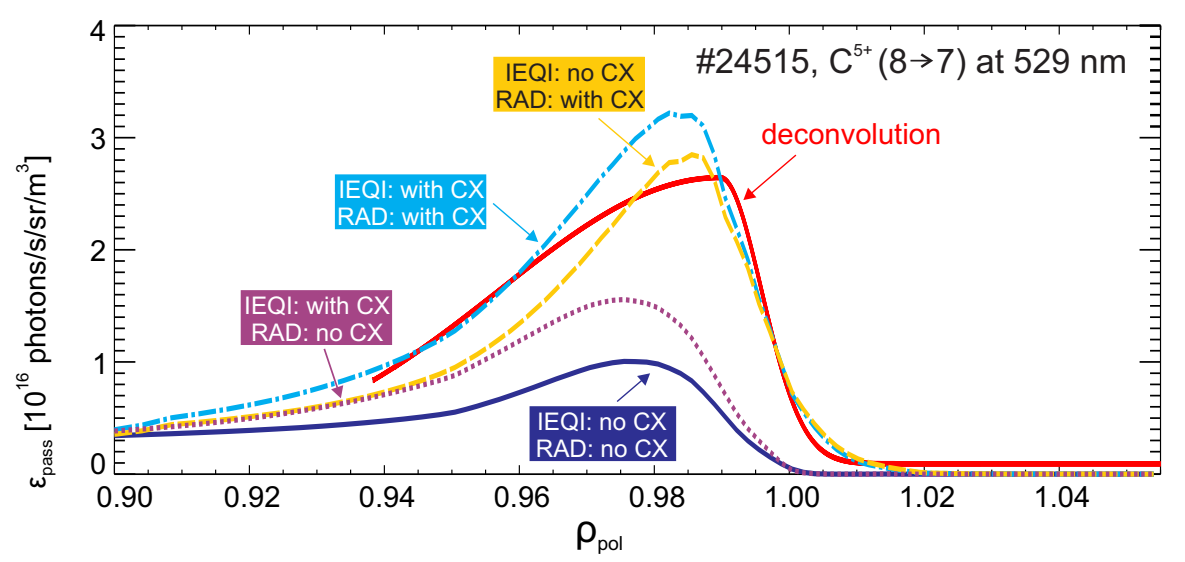

Figure 8. Local emissivity profiles of $\mathrm{C}^{5+}$ resulting from thermal $\mathrm{CX}$ (the label IEQI represents the ionization equilibrium, while radiation is abbreviated to RAD): deconvolution in red, STRAHL simulation in dark blue represents radiation due to electron impact excitation only; purple (dotted line) includes the effect on the ionization balance through CX recombination; in yellow (dashed line) the radiation due to local CX reactions with thermal neutral D is illustrated (here the ionization equilibrium has not been modified by CX recombination); light blue (chain line) accounts for electron impact excitation and both thermal CX effects. Note that in these simulations the local $\mathrm{n}_{D}$ has been scaled down by 2 , while the global $\mathrm{n}_{D}$ has been divided by 10 . 
8. The profile in purple shows the additional contribution of $\mathrm{CX}$ recombination on the ionization balance. As the maximal fractional abundance of $\mathrm{C}^{5+}$ is slightly shifted to lower $\rho_{\text {pol }}$ values, the maximum of the emissivity is also shifted. The profile in yellow shows the effect of radiation due to local CX reactions. For this simulation the PEC-CX have been taken into account and the ionization balance has not been changed through CX recombination. Note that direct excitation due to local CX processes provides for the steep gradient near the separatrix. However, the decay further inwards at $\rho_{\text {pol }} \approx 0.98$ is too strong and does not explain the long decay of the measured data. A combination of both CX effects is required. In figure 8 the simulated radiation in light blue illustrates the emissions when all CX effects are included. It should be noted that a different scaling factor has been used for both $\mathrm{n}_{D}$ profiles in order to obtain the profile which matches the measured data best. The local $n_{D}$ profile has been divided by a factor of 2 , while the global $n_{D}$ profile has been scaled down by a factor of 10 . Note that the scaling of $\mathrm{n}_{D}$ does not result in the same scaling of the local emission since both CX contributions have a different effect on the emissivity. In particular, both effects yield a distinct maximum of emission at different radial positions.

\subsection{Effect of transport on passive edge emission}

The effect of transport on the passive emission line of $\mathrm{C}^{5+}$ has been studied by increasing the transport coefficient profiles (drift velocity $\mathrm{v}$ and diffusion coefficient D) by a factor of 2. A scaling of the passive $\mathrm{C}^{5+}$ emission with the $\mathrm{C}^{6+}$ density at the maximum of the ETB, i.e. at the pedestal top, is found. Note that the pedestal top of the $\mathrm{C}^{6+}$ density is fixed as it is measured via active CXRS. The intensity of the passive emission is found to increase when the density gradient of $\mathrm{C}^{6+}$ flattens and accordingly, it decreases when the gradient is steeper. The density gradient is influenced by the ratio $\frac{v}{D}$ (which results from the transport analysis of the active CXRS measurements and has relatively small uncertainties) and thus, the local emissivity depends strongly on this parameter.

A rise in diffusion provokes an intensity reduction of the $\mathrm{C}^{5+}$ emissivity, but it also reduces the abundance of $\mathrm{C}^{6+}$ at the pedestal top and flattens the density gradient of $\mathrm{C}^{6+}$. Increasing the inward drift on the other hand results in a higher $\mathrm{C}^{5+}$ emission as well as in a higher $\mathrm{C}^{6+}$ density at the top of the ETB and in a steeper $\mathrm{C}^{6+}$ density gradient. The simultaneous change of both transport coefficients has a marginal effect, it slightly increases the emissivity of $\mathrm{C}^{5+}$ and the density of $\mathrm{C}^{6+}$, while the gradient of the $\mathrm{C}^{6+}$ density is almost unaffected. Figure 9 a) illustrates the effect of an increased diffusion (dotted line) and drift velocity (dashed line) on the passive emission line of $\mathrm{C}^{5+}$, respectively. In addition, the simulations employing the original transport coefficient profiles is shown (full lines). Note that the dark blue lines in figure 9 represent electron impact excitation processes, while the red curves result from simulations which also account for thermal CX. Increasing the diffusion coefficient leads to a stronger gradient for $\rho_{p o l} \leq 0.98$ and electron impact excitation (dotted dark blue line) would better describe the measurements. However, a rise in diffusion also affects the shape of the 

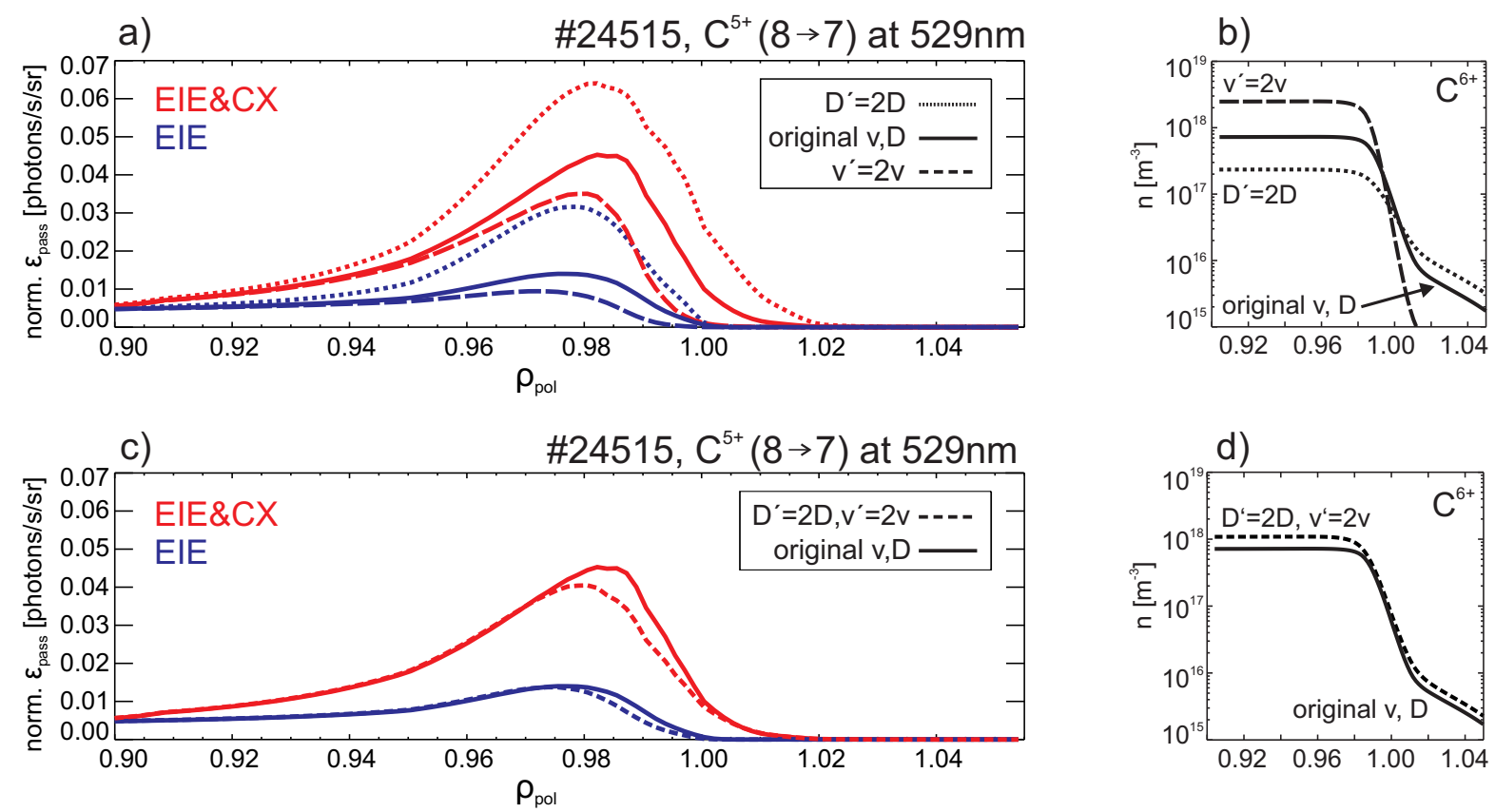

Figure 9. Effect of transport on the emissivity of $\mathrm{C}^{5+}$ : a) the simulated local emission (normalized to the value of the $\mathrm{C}^{6+}$ density at the pedestal top) employing the original transport coefficient profiles is represented by full lines, dotted lines illustrate the effect of an increased diffusion, while dashed lines show the effect of a higher inward drift; b) corresponding simulated $\mathrm{C}^{6+}$ density profiles; c) emissivity of $\mathrm{C}^{5+}$ when both transport coefficient profiles are changed (dashed lines) and using the original values (full lines); d) effect of increased diffusion and drift on the abundance of $\mathrm{C}^{6+}$.

density gradient of $\mathrm{C}^{6+}$. The gradient is flatter and does not fit to the measured $\mathrm{C}^{6+}$ density profile. In figure $9 \mathrm{~b}$ ) the corresponding effects of transport on the density of $\mathrm{C}^{6+}$ are illustrated. Figure 9 c) shows the carbon emissivity if both transport coefficients are changed simultaneously (dashed line), while figure $9 \mathrm{~d}$ ) shows the modified abundance of $\mathrm{C}^{6+}$. Note that in figure 9 a) and c) the passive emission has been normalized to the density of $\mathrm{C}^{6+}$ at the top of the ETB since the local emissivity profile of $\mathrm{C}^{5+}$ scales with the density of $\mathrm{C}^{6+}$ at the pedestal top.

\section{Modelling synthetic spectra}

Simulating synthetic spectra yields information on the spectral shape of the modelled passive line, which can directly be compared to the shape of the measured spectral line emitted at the plasma edge. This comparison enhances the quantification of both passive line components and benchmarks the developed forward model.

Virtual spectra can be calculated using the modelled emissivity profile and the ion temperature $\mathrm{T}_{i}$ and velocity profiles derived from the active CXRS measurements. The Doppler shift $\Delta \lambda$ of an emission line results from the toroidal rotation $\mathrm{v}_{t o r}$ and yields information on the central wavelength of the emission line, while the ion temperature gives the Doppler width of the spectral line. The effective central wavelength of the 
passive $\mathrm{C}^{5+}$ emission differs from that of the active component by $3 \mathrm{pm}$ [33] if the $l$ distribution of the electron, which is captured by the impurity ion during a CX collision, is different. The electron can be transferred between different $l$ levels before dropping to lower states via photon radiation causing a redistribution of the $l$ states. These $l$-mixing processes disturb the relative populations between the excited levels in a certain $\mathrm{n}$ state and have an impact on the line intensity profiles. For the passive emission of $\mathrm{C}^{5+}$, the main contribution to all thermal CX processes is given by thermal neutral $D$ in $n=2$.

In order to treat the $l$-mixing problem accurately, an empirical formula for determining the critical plasma density at which a given transition is fully mixed has been provided by [34]:

$$
n_{e} \geq \frac{1}{8.5 \cdot 10^{-16}} \sqrt{\frac{Z^{15}}{n^{17}}}
$$

$\mathrm{n}$ is the principal quantum number, $\mathrm{Z}$ the charge of the ion and $\mathrm{n}_{e}$ the electron density in $\mathrm{cm}^{-3}$. For the $\mathrm{C}^{5+}(\mathrm{n}=8 \rightarrow 7)$ spectral line a critical density of $1.7 \cdot 10^{18} \mathrm{~m}^{-3}$ is obtained which is clearly lower than the typical densities of $10^{19}$ to $10^{20} \mathrm{~m}^{-3}$ at the edge pedestal of ASDEX Upgrade. This indicates that for laboratory plasmas at ASDEX Upgrade the $l$-levels are completely mixed. Hence, the $l$-distribution of the electrons is assumed to be the same for both the active and the passive component.

Figure 10 shows the measured $\mathrm{T}_{i}$ and $\mathrm{v}_{\text {tor }}$ profiles derived from a discharge which has the same setup as the one analyzed for the passive carbon emission. Since no measured data are available in the SOL, an exponential decay was assumed for the toroidal plasma rotation and $\mathrm{T}_{i}$ has been extrapolated for $\rho_{\text {pol }}>1.0$. The spectral radiance at each step
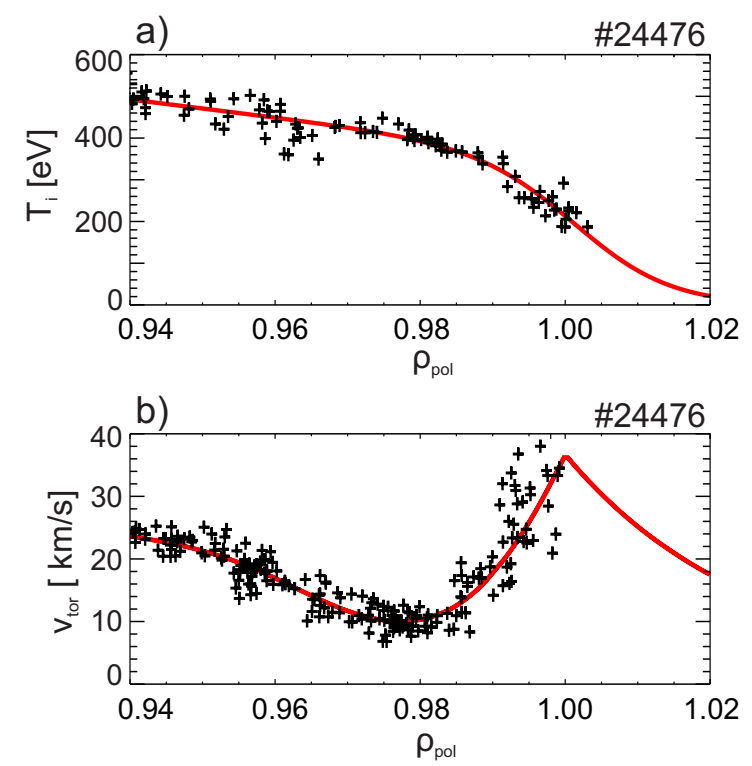

Figure 10. Input profiles for simulating synthetic spectra obtained from active CXRS: a) Measurements of $\mathrm{T}_{i}$ in black, modelled $\mathrm{T}_{i}$ profile (red) fitted to experimental data: for $\rho_{p o l}>1.0 \mathrm{~T}_{i}$ has been extrapolated; b) measured $\mathrm{v}_{t o r}$ profile in black, for modelling $\mathrm{v}_{\text {tor }}$ (red) the profile inside the separatrix has been fitted to match the measurements, while an exponential decay has been assumed in the SOL. 
along the LOS is represented by a Gaussian function and by summing up the intensities of all steps, which just corresponds to an integration along the viewing chord, the line-integrated intensity of the spectral line can be derived. Synthetic spectra have been modelled for $\mathrm{C}^{5+}$ using the local emissivity profile predicted by STRAHL and implementing the deconvoluted radial profile which results from the forward model. In figure 11 a) a typical line-integrated passive spectrum detected by the edge CXRS system in the ETB at $\rho_{\text {pol,min }} \approx 0.94$ and in figure $11 \mathrm{~b}$ ) a passive spectrum detected near the separatrix are illustrated, respectively. At ASDEX Upgrade, the measured CX spectra are fitted by the routine CXSFIT [35]. Using this code, the passive component can usually be described by a single Gaussian-shaped function which represents a small, line-integrated emission in the ETB close to the separatrix. The single Gaussian fit to the measured passive spectrum in figure 11 a) yields an apparent $\mathrm{T}_{i}$ of $379 \mathrm{eV}$, while for the spectrum in figure $11 \mathrm{~b}$ ) the code predicts an apparent $\mathrm{T}_{i}$ value of $190 \mathrm{eV}$. The red synthetic spectra of figure 11 result from implementing the local emissivity profile determined by forward modelling, while in blue the radiation predicted by STRAHL has been included. The input emissivity profile modelled with STRAHL accounts for both electron impact excitation and thermal CX. Note that the STRAHL simulation, which employs a different scaling of both $\mathrm{n}_{D}$ profiles, is used for calculating the synthetic spectra in light blue in figure 11. A background emission (constant in $\lambda$ ) of $2.0 \cdot 10^{16}$ $\frac{\text { photons }}{\mathrm{m}^{2} \mathrm{sr} \mathrm{nm} \mathrm{s}}$ and $1.0 \cdot 10^{16} \frac{\text { photons }}{\mathrm{m}^{2} \mathrm{sr} \mathrm{nm} \mathrm{s}}$ has been added to the simulated spectral radiance
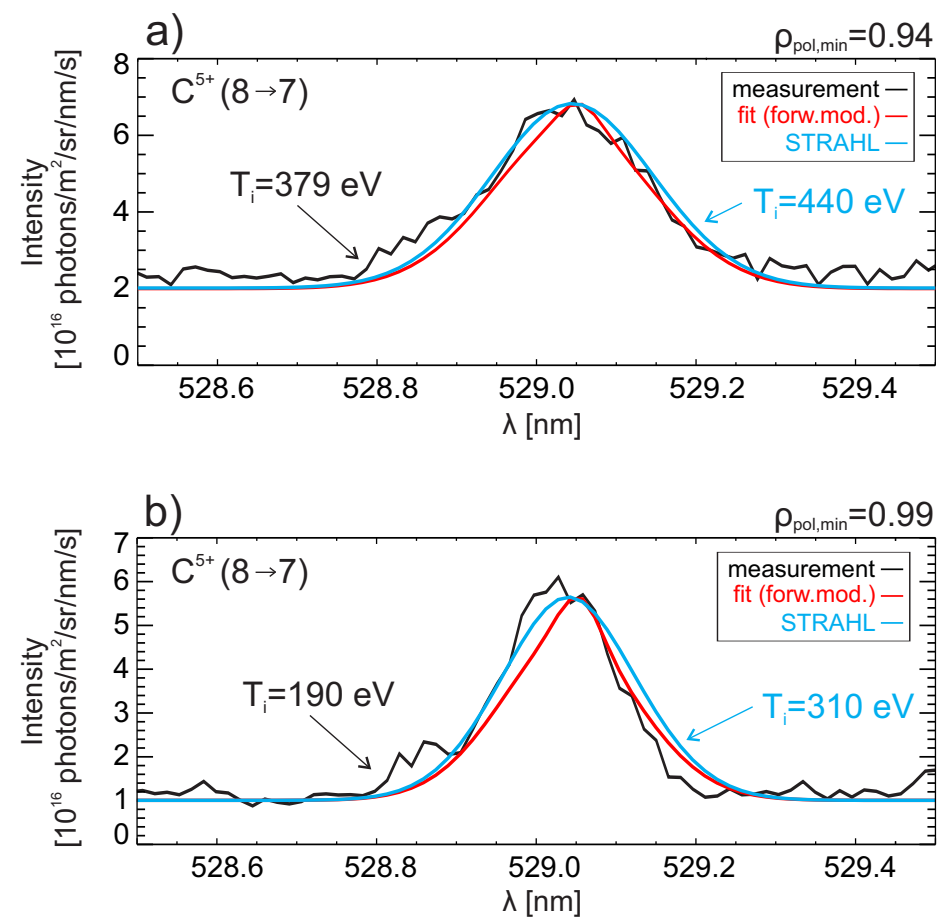

Figure 11. Reconstruction of measured passive spectra: a) spectrum detected at $\rho_{\text {pol,min }} \approx 0.94$, b) spectrum recorded near last closed flux surface; measured spectra in black, synthetic spectra resulting from forward model in red and in blue virtual spectra modelled with STRAHL radiation including both passive components. 
in figure 11 a) and b), respectively, in order to account for the background level of the measured line-integrated spectrum.

For comparing the shape of the spectra, the spectral radiance has been scaled to fit the maximum of measured intensity. In figure 11 a) the light blue spectrum, which represents both components of the passive carbon emission, has been scaled by a factor of 0.95. This factor results from the uncertainties of $\mathrm{T}_{i}$ and $\mathrm{v}_{t o r}$ in the SOL. The spectrum which employs the local emissivity profile modelled by STRAHL yields a slightly higher Doppler width, i.e. a higher value for $\mathrm{T}_{i}$. The red spectrum resulting from the modified radial Gaussian provides for an ion temperature of approximately $350 \mathrm{eV}$, which agrees well with the $\mathrm{T}_{i}$ value obtained with CXSFIT. The virtual spectrum resulting from the simulation which combines electron impact excitation and thermal CX provides for $\mathrm{T}_{i}$ $\approx 440 \mathrm{eV}$. In figure $11 \mathrm{~b}$ ) the light blue spectrum has been multiplied by a factor of 1.4 . Note that the scaling factor differs since there is more relative contribution of the SOL values, which might be overestimated. The simulated spectra provide a slightly higher Doppler width than obtained with CXSFIT. The synthetic spectrum resulting from the modified radial Gaussian function (red spectral profile in figure $11 \mathrm{~b}$ ) at $\rho_{\text {pol,min }} \approx 0.99$ ) implies an ion temperature of $\mathrm{T}_{i} \approx 225 \mathrm{eV}$, while the light blue spectrum (electron impact excitation and thermal $\mathrm{CX}$ ) yields $\mathrm{T}_{i} \approx 310 \mathrm{eV}$.

Comparing the $\mathrm{T}_{i}$ value of the virtual spectra (for both cases in figure 11), which are obtained using the emissivity profile predicted by STRAHL, to the $\mathrm{T}_{i}$ value of the measured passive spectra yields a difference of approximately $100-150 \mathrm{eV}$. Note that the measured spectrum has been fitted with only one Gaussian leading to a lower value for $\mathrm{T}_{i}$. It should be noted that the $\mathrm{T}_{i}$ and $\mathrm{v}_{\text {tor }}$ values are taken from beam based CXRS measurements employing $\mathrm{C}^{6+}$, while the passive components are emitted by different subspecies of carbon which exhibit different local pressure gradients. These effects are difficult to be disentangled unambiguously and thus, this route was not pursued further.

The separation of both passive components in the measured $\mathrm{C}^{5+}$ spectra is not possible since the spectral line appears as a sole component which can be fitted by one Gaussian function. Photons which are emitted from the recombined ion after CX originate typically from ions which have a higher temperature than those which emit photons after electron impact. Thus, the spectral line would show a stronger Doppler broadening and possibly a different Doppler shift. However, this distinction is hardly visible in the forward modelling of the passive spectra due to the fact that the contribution from the hotter regions plays only a relatively small role in the lineintegrated total signal.

\section{Summary and Conclusions}

The derivation of ion density, temperature and velocity profiles requires the distinction between active and passive signal in a superimposed complex CX spectrum. Therefore, accurate modelling and understanding of the passive component is indispensable. In this work, the local emissivity of the passive CX radiation has been calculated from the 
measurements. The deconvoluted local emissivity profile has been compared with the simulated radiation obtained with the 1-D impurity transport code STRAHL allowing to quantify the contributions from electron impact excitation and thermal CX, respectively. For simulating thermal CX, knowledge of the penetration of the background neutrals is required. A local and a flux surface averaged neutral D density profile are needed and have been derived from modelling using the kinetic transport algorithm KN1D. The analysis of the passive edge emission of $\mathrm{C}^{5+}$ yields that both electron impact excitation and thermal CX contribute. This is consistent with the findings of previous studies such as $[4,5,6,7]$. Indeed, it is found that thermal $\mathrm{CX}$ is the dominant effect. It yields a strong contribution to the passive $\mathrm{C}^{5+}$ emission via two mechanisms, i.e. CX recombination and radiation due to local CX processes. Studying the effect of transport on the local emissivity profile of $\mathrm{C}^{5+}$ yields that the intensity of the passive $\mathrm{C}^{5+}$ emission scales with the density of fully ionized $\mathrm{C}^{6+}$ at the pedestal top. In particular, the intensity of the local emissivity profile depends on the ratio of the transport coefficients $\frac{v}{D}$, which is specified by the measured $\mathrm{C}^{6+}$ density.

The measured passive spectra of $\mathrm{C}^{5+}$ can be described by one single Gaussian. Hence, it is difficult to separate both passive components in the measured line-integrated spectra even though the CX emission profile has its maximum further outside compared to the maximum of the electron impact emission. The synthetic spectra describe the measured spectra reasonably well, certifying the shape and the Doppler width of the passive component. The passive line is a superposition of several Gaussian line shapes with different temperatures and Doppler shifts. For the innermost chord it can be fitted by a single Gaussian with temperatures in the range of approximately 300 to $400 \mathrm{eV}$.

The developed forward model and the high spatial resolution of the edge CXRS diagnostic at ASDEX Upgrade allow a deconvolution of the line-integrated measurements and thus, to obtain a radial emissivity profile of the passive component. The presented work is a first step for a quantitative characterization of the passive emission line independent of beam modulation. For ASDEX Upgrade conditions the emission is dominated by thermal CX with recycling D neutrals. Hence, their local influx as well as their flux surface averaged influx, i.e. the global penetration of the neutrals, need to be known. The derived $\mathrm{n}_{D}$ profiles overestimate the measured photon fluxes of $\mathrm{C}^{5+}$. Ionization gauge measurements in a remote location seem not to be

sufficient. Further gauge measurements combined with an improved edge modelling of $\mathrm{n}_{D}$ are required to provide a more comprehensive picture of the distribution of the background neutrals.

\section{References}

[1] R.J. Fonck et al. Determination of plasma-ion velocity distribution via charge-exchange recombination spectroscopy. Phys. Rev. A, 29(6), 1984.

[2] R.C. Isler. A review of charge-exchange spectroscopy and applications to fusion plasmas. Physica Scripta, 35:650, 1987.

[3] M. G. von Hellermann et al. Complex spectra in fusion plasmas. Physica Scripta, T120:19, 2005. 
[4] M. Tunklev et al. Modelling of passive charge exchange emission and neutral background density deduction in JET. Plasma Phys. Control. Fusion, 41:985, 1999.

[5] C. F. Maggi et al. Impurity Line Emission due to Thermal Charge Exchange in JET Edge Plasmas. Conf. Proceedings of the $21^{\text {st }}$ EPS Conference on Plasma Physics, Montpellier, 1994.

[6] A. Boileau et al. JET Joint Undertaking Report JET-P(87)44, 1987.

[7] E. J. Synakowski et al. A model for edge spectral line emission and application to ion temperature measurements with charge-exchange recombination spectroscopy. Rev. Sci. Instrum., 66(1):649, 1995.

[8] F. Wagner et al. Regime of Improved Confinement and High Beta in Neutral-Beam-Heated Divertor Discharges of the ASDEX Tokamak. Phys. Rev. Lett., 49(19):1408, 1982.

[9] A. Herrmann et al. Special Issue on ASDEX Upgrade. Fusion Science and Technology Special Issue, 44(3), 2003.

[10] T. Pütterich et al. Fast CXRS-Measurements in the Edge Transport Barrier of ASDEX Upgrade. Conf. Proceedings of the $35^{\text {th }}$ EPS Conference on Plasma Physics, Crete, 2008.

[11] T. Pütterich et al. Evidence for Strong Inversed Shear of Toroidal Rotation at the Edge-Transport Barrier in ASDEX Upgrade. Phys. Rev. Lett., 102(025001), 2009.

[12] M. G. von Hellermann et al. Status of the DNB based ITER CXRS and BES diagnostic. Rev. Sci. Instr., 77, 2006.

[13] K. H. Behringer. Description of the impurity transport code STRAHL. JET Report JET-R(87)08, 1987.

[14] R. Dux. STRAHL User Manual. IPP Report 10/30, Max-Planck-Institute for Plasma Physics, Garching, Germany, 2006.

[15] W. Suttrop. Practical Limitations to Plasma Edge Electron Temperature Measurements by Radiometry of Electron Cyclotron Emission. IPP Report 1/306, Max-Planck-Institute for Plasma Physics, Garching, Germany, 1997.

[16] H. Murmann et al. The Thomson scattering systems of the ASDEX Upgrade tokamak. Rev. Sci. Instrum., 63:4941, 1992.

[17] E. Wolfrum et al. Fast lithium-beam spectroscopy of tokamak edge plasmas. Rev. Sci. Instrum., 64(8):2285, 1993.

[18] R. Fischer et al. Probabilistic lithium beam data analysis. Plasma Phys. Control. Fusion, 50:085009, 2008.

[19] O. Gehre. The HCN-laser-interferometer of the divertor tokamak ASDEX. International Journal of Infrared and Millimeter Waves, 5(3), 1984.

[20] P. C. Stangeby. The Plasma Boundary of Magnetic Fusion Devices. Institute of Physics Publishing, 2000.

[21] H. Zohm. Edge Localized Modes (ELMs). Plasma Phys. Control. Fusion, 38(2):105, 1996.

[22] T. Pütterich et al. ELM Flushing and Impurity Transport in the H-mode Edge Barrier in ASDEX Upgrade. J. Nucl. Mater. (submitted), 2010.

[23] H. P. Summers. ADAS User Manual 2.6. http://www.adas.ac.uk/manual.php, 2004.

[24] R. Hoekstra et al. Charge exchange from $\mathrm{D}(\mathrm{n}=2)$ atoms to low- $\mathrm{Z}$ receiver ions. Plasma Phys. Control. Fusion, 40:1541, 1998.

[25] W. L. Wiese et al. Atomic Transition Probabilities: H through Ne - A Critical Data Compilation, volume 1. Natl. Stand. Ref. Data Ser., Natl. Bur. Stand. (U.S.), NSRDS-NBS 4, 1966.

[26] Ph. Mertens et al. Hydrogen release from plasma-facing components into fusion plasmas - recent results from a spectroscopic approach. Plasma Phys. Control. Fusion, 43:A349, 2001.

[27] J. Harhausen. Interpretation of $D_{\alpha}$ Imaging Diagnostics Data on the ASDEX Upgrade Tokamak. $\mathrm{PhD}$ thesis, Ludwig-Maximilian University Munich, 2009.

[28] K. McCormick et al. Main chamber high recycling on ASDEX Upgrade. Journ. Nucl. Materials, 390:465, 2009.

[29] B. LaBombard. KN1D User Manual. http://www.psfc.mit.edu/ labombard/KN1D_Source_Info.html, 2001. 
[30] A. Kallenbach et al. The influence of spatially and temporally varying edge conditions on the interpretation of spectroscopic flux measurements. Plasma Phys. Control. Fusion, 36:1299, 1994.

[31] A. Pospieszczyk. Diagnostics of edge plasma by optical methods. Atomic and Plasma-Material Interaction Processes in Controlled Thermonuclear Fusion, ed R. K. Janev and H. W. Drawin, Elsevier, Amsterdam, 1993.

[32] K. H. Behringer. Spectroscopic studies of plasma-wall interaction and impurity behaviour in tokamaks. J. Nucl. Mater., 145-147:145, 1987.

[33] K. Ida et al. Measurements of poloidal rotation velocity using charge exchange spectroscopy in a large helical device. Rev. Sci. Instrum., 71(6):2360, 2000.

[34] D. H. Sampson. On statistical equilibrium among the sublevels of hydrogenic atoms and ions. $J$. Phys. B: At. Mol. Phys., 10(4):749, 1977.

[35] A. D. Whiteford et al. CXSFIT User Manual. http://www.adas.ac.uk/notes/adas_r07-01.pdf, 2007. 